\title{
Efficacy and safety of alirocumab in Korean patients with hypercholesterolemia and high cardiovascular risk: subanalysis of the ODYSSEY-KT study
}

\author{
Chang-Wook Nam ${ }^{1}$, Dong-Soo Kim² ${ }^{2}$ Jianyong $\mathrm{Li}^{3}$, Marie T. Baccara-Dinet ${ }^{4}$, Ivy Li ${ }^{3}$, Ji-Hyun Kim ${ }^{5}$ and \\ Chong-Jin $\mathrm{Kim}^{6}$
}

\begin{abstract}
${ }^{1}$ Division of Cardiology, Department of Internal Medicine, Keimyung University Dongsan Medical Center, Daegu; ${ }^{2}$ Department of Internal Medicine, Inje University Busan Paik Hospital, Busan, Korea; ${ }^{3}$ Sanofi R\&D, Shanghai, China; ${ }^{4}$ Sanofi R\&D, Montpellier, France; ${ }^{5}$ Sanofi Korea, Seoul; ${ }^{6}$ Department of Internal Medicine, Kyung Hee University Hospital at Gangdong, Seoul, Korea
\end{abstract}

Received: April 9, 2018

Revised : April 26, 2018

Accepted: April 30, 2018

Correspondence to

Chong-Jin Kim, M.D.

Department of Internal Medicine, Kyung Hee University Hospital at Gangdong, 892 Dongnam-ro, Gangdong-gu, Seoul 05278, Korea

Tel: +82-2-440-6106

Fax: +82-2-440-7699

E-mail: Chongjinkim@naver.com

Chang-Wook Nam, M.D.

Division of Cardiology, Department of Internal Medicine, Keimyung University Dongsan Medical Center, 56 Dalseong-ro, Jung-gu, Daegu 41931, Korea

Tel: +82-53-250-8015

Fax: $+82-53-250-7034$

E-mail: ncwcv@dsmc.or.kr
Background/Aims: Efficacy and safety data of alirocumab, a fully human monoclonal antibody to proprotein convertase subtilisin/kexin type 9 (PCSK9), is not yet well established in the Korean population. We assessed them in ODYSSEY-KT through the pre-specified Korean subanalysis.

Methods: In the ODYSSEY-KT study, South Korean and Taiwanese patients with hypercholesterolemia and high cardiovascular risks were randomized (1:1) to alirocumab or placebo. Alirocumab was self-administered subcutaneously at $75 \mathrm{mg}$ every 2 weeks with a maximally tolerated statin dose with or without other lipid-modifying therapies. Alirocumab dose was increased to $150 \mathrm{mg}$ every 2 weeks at week 12 if low density lipoprotein cholesterol (LDL-C) $\geq 70 \mathrm{mg} / \mathrm{dL}$ at week 8 . Primary endpoint was percent change in LDL-C from baseline to week 24. Results from Korean cohort $(n=83: 40$ for alirocumab and 43 for placebo, respectively) analyses are reported here.

Results: In alirocumab group, the least square of mean change percent in LDL-C levels was $-65.7 \%$ (placebo: 11.1\%; $p<0.0001$ ) and 92.0\% of them achieved LDL-C $<70 \mathrm{mg} / \mathrm{dL}$ (placebo: 12.7\%; $p<0.0001$ ) at week 24. Alirocumab also showed significantly greater improvements in high density lipoprotein cholesterol (HDL-C), non-HDL-C, total cholesterol, lipoprotein(a), and apolipoprotein B than placebo $(p<0.05)$. Two consecutive calculated LDL-C values $<25 \mathrm{mg} / \mathrm{dL}$ were observed in $37.5 \%$ of alirocumab-treated patients. Overall, $45.0 \%$ alirocumab-treated and $51.2 \%$ placebo-treated patients experienced treatment-emergent adverse events (TEAEs) without discontinuation of treatment due to TEAEs.

Conclusions: Alirocumab has demonstrated to be effective in improvement of LDL-C and related lipid profiles in Korean cohort. Alirocumab was generally well tolerated with no significant safety signals.

Keywords: PCSK9; Cholesterol, LDL; ODYSSEY; Hypercholesterolemia

\section{INTRODUCTION}

Dyslipidemia is a major risk factor for cardiovascular disease (CVD), the second-leading cause of mortality in
Korea [1-3] and is also associated with increased prevalence of metabolic syndrome. In recent years, the prevalence of dyslipidemia has been increasing steadily in Korea $[4,5]$. The association between higher low density 
lipoprotein cholesterol (LDL-C) level and CVD is well established and guidelines recommend $<70$ or $<100$ $\mathrm{mg} / \mathrm{dL}$ as target levels in patients with very high or high CV risk, respectively $[6,7]$.

Guidelines for the management of dyslipidemia in Korea were established in 2015 [8]. These guidelines recommend lifestyle modification and pharmacological management of dyslipidemia for prevention of CVD. The CEPHEUS Pan-Asian survey showed suboptimal achievement of target LDL-C and total cholesterol (TC) with existing treatments in Korea [9].

Among several lipid lowering therapies (LLTs), proprotein convertase subtilisin/kexin type 9 ( $\mathrm{PCSK}_{9}$ ) inhibitors modulate cholesterol homeostasis via its ability to upregulate LDL receptor expression on hepatocyte cell membrane and by decreasing plasma LDL-C levels [10]. Recent consensus statements from both the American College of Cardiology and European Society of Cardiology/European Atherosclerosis Society recommend the use of PCSK9 inhibitors or ezetimibe in patients with very high or high cardiovascular (CV) risk who have high baseline LDL-C levels (70 to $189 \mathrm{mg} / \mathrm{dL}$ ) uncontrolled by maximally tolerated doses of statins $[11,12]$. Alirocumab is a fully human monoclonal antibody that inhibits PCSK9 and has been clinically evaluated for its ability to reduce LDL-C levels. Several ODYSSEY phase 3 studies have demonstrated that alirocumab can reduce LDL-C levels substantially when used as monotherapy or when combined with background statin therapy with or without other LLTs [13-21].

However, the safety and efficacy of alirocumab has not been established in the Korean population. The ODYSSEY KT study was performed to assess the safety and efficacy of alirocumab among Korean and Taiwanese patients with uncontrolled serum cholesterol levels and coronary heart disease (CHD) or those at risk for CHD [22]. Here we present a pre-specified subanalysis for Korean patients.

\section{METHODS}

\section{Study design}

The ODYSSEY KT study (NCTo2289963) was a randomized, double-blind, placebo-controlled, parallel-group, and multicenter study [22]. The Korean cohort study was conducted in 16 study centers across Korea between January 2015 and April 2016. The protocol was approved by the relevant independent Institutional Review Boards (Keimyung University Dongsan Medical Center IRB 2014-09-009). The study was performed according to the principles of the Declaration of Helsinki and applicable amendments and the International Conference on Harmonisation guidelines for Good Clinical Practice. All participating patients provided written informed consent.

\section{Patients}

Eligible patients (aged $\geq 18$ years) had established CHD or CHD risk equivalents and hypercholesterolemia that was inadequately controlled with a maximally tolerated statin dose (with or without other LLTs) at a stable dose $\geq 4$ weeks prior to screening. CHD risk equivalents were defined as history of CVD, moderate chronic kidney disease, or diabetes with multiple risk factors. Inadequately controlled hypercholesterolemia was defined as LDL-C $\geq 70 \mathrm{mg} / \mathrm{dL}$ in patients with documented CVD history or $\geq 100 \mathrm{mg} / \mathrm{dL}$ in patients without CVD history. The following were considered maximally tolerated statin doses: atorvastatin, 40 to $80 \mathrm{mg} /$ day; rosuvastatin, $20 \mathrm{mg} /$ day; and simvastatin, $40 \mathrm{mg} /$ day. Patients receiving daily dose of atorvastatin, rosuvastatin, or simvastatin dose and considered appropriate by the investigator were also eligible to participate. Exclusion criteria for this study are described in Supplementary Table 1.

\section{Treatment regimen}

The total study duration was 35 weeks, consisting of a 3-week screening period, 24 weeks of double-blind treatment, and an 8-week follow-up period. Eligible patients were randomized 1:1 to receive either alirocumab $75 \mathrm{mg}$ every 2 weeks or placebo every 2 weeks using an auto-injector device through the subcutaneous route. Study visits were on weeks $0,4,8,12,16$, and 24. Follow visit was done 8 weeks after the end of treatment visit. Patients on alirocumab who did not achieve LDL-C $<70 \mathrm{mg} / \mathrm{dL}$ at week 8 were uptitrated to alirocumab $150 \mathrm{mg}$ every 2 weeks from week 12 onwards. Blinding was maintained in these patients.

\section{Endpoints}

The primary efficacy endpoint was the percent change 
in calculated LDL-C values between baseline and week 24 in the intent-to-treat (ITT) population. Other key endpoints included the percent change from baseline to week 24 in non-high density lipoprotein cholesterol (non-HDL-C), TC, HDL-C, fasting triglycerides (TGs), lipoprotein(a)(Lp(a)), apolipoprotein A1 and apolipoprotein $\mathrm{B}$ (Apo B) and the proportion of patients reaching a calculated LDL-C $<70 \mathrm{mg} / \mathrm{dL}$ at week 24. Analyses of lipid samples were performed by a central laboratory using standard procedures and individual site laboratory tests for lipid parameters were not allowed during the study period. LDL-C levels were calculated using the Friedewald formula. LDL-C levels were reflexively measured via $\beta$-quantification when TG levels were $>400 \mathrm{mg} / \mathrm{dL}$. In addition, LDL-C levels were systematically measured (via the $\beta$-quantification method) at weeks o and 24 for efficacy analysis purposes.

Safety was assessed throughout the study by monitoring treatment-emergent adverse events (TEAEs), serious adverse events (SAEs), laboratory data, and vital signs. TEAEs were defined as adverse events (AEs) that occurred during the time from first administration of blinded study drug to 10 weeks after last administration of blinded study drug.

\section{Statistical analysis}

The sample size calculation has been described previously [22]. Briefly, a total sample size of 40 patients (20 patients in each group) was required to achieve $95 \%$ power to detect a mean percent change difference in LDL-C of 30\% with a 0.05 two-sided significance level and assuming a common standard deviation (SD) of $25 \%$ with all patients having an evaluable primary endpoint. At last, total sample size $(n=83)$ was bigger than the estimated minimum size to satisfy the requirement because we also intended to identify the safety data as well as efficacy. Calculations were done using nQuery Advisor version 7.0 (Statsols, Cork, Ireland).

The primary efficacy endpoint was analyzed using a mixed effect model with repeated measures (MMRM) with all post-baseline data available and missing data accounted for. Fixed categorical effects of treatment group (alirocumab vs. placebo), time (weeks 4, 8, 12, 16, and 24), randomization strata, treatment-by-time point interaction, strata-by-time point interaction, and continuous fixed covariates of baseline LDL-C value and baseline value-by-time point interaction were included in the MMRM. The 95\% confidence intervals (CIs) of the differences were estimated from the model.

Other key endpoints were analyzed in a sequential order using a hierarchical inferential approach when the primary endpoint was significant at $5 \% \alpha$ level. Continuous secondary endpoints with an anticipated normal distribution were analyzed using MMRM. Continuous secondary endpoints with a normal distribution (lipids other than Lp(a) and TGs) were analyzed as for the primary endpoint while those without normal distribution (Lp(a) and TGs) and binary secondary endpoints were analyzed using multiple imputation approach followed by robust regression. Target achievement for LDL-C was analyzed by multiple imputations followed by logistic regression. The ITT population included all randomized patients with LDL-C values available at baseline and at least one other post-randomization time point till the end of study. The modified intent-to-treat (mITT) included the randomized patients who had received at least one dose or part of the study with an evaluable primary endpoint during the treatment period and within one of the analysis windows. All randomized patients who received at least one dose or part of a dose of the alirocumab comprised the safety set. Safety data were analyzed by descriptive statistics and were focused on the TEAE period. All statistical analyses were conducted using SAS version 9.4 (SAS Institute Inc., Cary, NC, USA).

\section{RESULTS}

\section{Patient characteristics}

A total of 83 patients were enrolled in the Korean subgroup of the ODYSSEY KT study. Both the ITT and safety populations consisted of 83 patients (40 patients in the alirocumab group and 43 patients in the placebo group) while the mITT population comprised 82 patients (40 patients in the alirocumab group and 42 patients in the placebo group) (Fig. 1). Both treatment groups were matched for age, gender, weight, body mass index, medical history, and statin type by intensity. More patients in alirocumab group were taking ezetimibe than those in placebo group ( $n=7,17.5 \%$; and $n=4,9.3 \%$, respectively), but it did not reach the significance level of 0.05 
in the post hoc chi-square test (Table 1). Additionally, no significant differences were found in lipid parameters between alirocumab and placebo groups at baseline $(p>$ 0.05; post hoc $t$ tests) (Table 2).

\section{Efficacy analyses}

There was a reduction in LDL-C levels at week 4 and it was maintained till week 24. The calculated least squares mean $(\mathrm{LSM}) \pm$ standard error $(\mathrm{SE})$ of LDL-C levels in the ITT population receiving alirocumab decreased from $90.7 \pm 3.6 \mathrm{mg} / \mathrm{dL}$ at baseline to $33.8 \pm 4.5 \mathrm{mg} / \mathrm{dL}$ at week 24 (Fig. 2). In comparison, LSM \pm SE of LDL-C levels increased from 95.1 \pm 4.1 at baseline to $102.9 \pm 4.4$ at week 24 in patients receiving placebo. The percent change in $\mathrm{LSM} \pm \mathrm{SE}$ of LDL-C from baseline to week 24 was $-65.7 \% \pm 4.4 \%$ in the alirocumab group in comparison with $11.1 \% \pm 4.2 \%$ ) in the placebo group. The difference in $\mathrm{LSM} \pm \mathrm{SE}$ for alirocumab versus placebo was $-76.7 \% \pm$ 6.1\% (95\% CI, -88.9 to -64.6 ; $p<0.0001$ ) (Table 3 and Fig. 3). Similarly, the percent change in LDL-C from baseline for the mIT'T population given as $\mathrm{LSM} \pm \mathrm{SE}$ was $-67.3 \%$ $\pm 4.4 \%$ in the alirocumab group in comparison with $11.1 \% \pm 4.2 \%$ in the placebo group and the difference in $\mathrm{LSM} \pm \mathrm{SE}$ for alirocumab versus placebo was $-78.5 \% \pm$ 6.1\% (95\% CI, -90.6 to -66.3 ; $p<0.0001$ ) (Fig. 4).

Significant differences in $\mathrm{LSM} \pm \mathrm{SE}$ for alirocumab versus placebo were observed at week 24 in the following lipid parameters: non-HDL-C $(-62.4 \% \pm 4.5 \%$; $95 \%$ CI, -71.4 to $-53.4 ; p<0.0001)$, HDL-C $(9.0 \% \pm 4.2 \% ; 95 \%$ CI, 0.7 to $17.4 ; p=0.034)$, TC $(-41.9 \% \pm 3.4 \% ; 95 \% \mathrm{CI},-48.6$ to $-35.3 ; p<0.0001), \operatorname{Lp}(\mathrm{a})(-40.9 \% \pm 7.7 \% ; 95 \% \mathrm{CI},-56.1$ to $-25.7 ; p<0.0001)$, and ApoB (-57.0\% $\pm 4.5 \%$; $95 \%$ CI, -66.0 to $-48.1 ; p<0.0001$ ) (Table 3 and Fig. 3). The same trend was found in mITT comparisons (Supplementary Table 2).

The proportion of patients achieving LDL-C $<70 \mathrm{mg} /$ $\mathrm{dL}$ at week 24 was $92.0 \%$ in the alirocumab group and $12.7 \%$ in the placebo group (combined estimate for odds ratio, $157.4 ; 95 \%$ CI, 19.0 to $1,306.7 ; p<0.0001)$. Three alirocumab-treated patients (7.5\%) had LDL-C values $\geq$ $70 \mathrm{mg} / \mathrm{dL}$ at week 8 and were uptitrated to alirocumab $150 \mathrm{mg}$ every 2 weeks at week 12. During the treatment period, 15 alirocumab-treated patients (37.5\%) had two consecutive calculated LDL-C values $<25 \mathrm{mg} / \mathrm{dL}$, among whom four patients (10.0\%) displayed two consecutive LDL-C values $<15 \mathrm{mg} / \mathrm{dL}$.

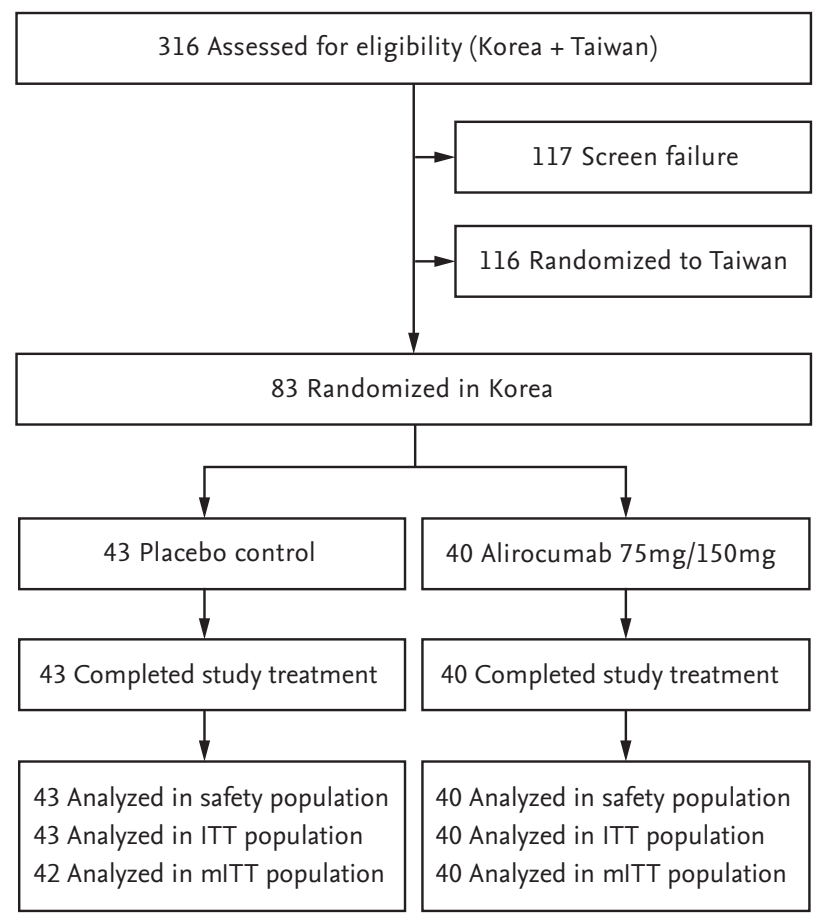

Figure 1. Patient flow: CONSORT diagram. ITT, intent-totreat; mITT, modified intent-to-treat.

\section{Safety analysis}

A total of 18 patients $(45.0 \%)$ in the alirocumab group and 22 patients (51.2\%) in the placebo group experienced a TEAE. The most commonly reported TEAEs in the alirocumab group by system organ class (SOC) and preferred term (PT) classification were nervous system disorders $(\mathrm{n}=5,12.5 \%)$, majority of which were headaches ( $n=4,10.0 \%)$; followed by infections and infestations ( $\mathrm{n}$ $=4,10.0 \%)$, majority of which were nasopharyngitis $(\mathrm{n}=$ 3, 7.5\%); and injury, poisoning, and procedural complications $(\mathrm{n}=4,10.0 \%)$, majority of which were due to fall $(\mathrm{n}=2,5.0 \%)$ (Table 4$)$. The most commonly reported TEAEs in placebo group by SOC and PT classification were infections and infestations ( $\mathrm{n}=6,14.0 \%)$, followed by gastrointestinal disorders $(\mathrm{n}=5,11.6 \%)$ and general disorders and administration site conditions $(\mathrm{n}=5,11.6 \%)$ (Table 4). Among alirocumab-treated patients who had two consecutive calculated LDL-C $<25 \mathrm{mg} / \mathrm{dL}(\mathrm{n}=15)$, one case of hepatobiliary disorders (hepatitis acute) and one case of injury, poisoning and procedural complications (fall and radius fracture for the same case) were identified. 
Table 1. Patient characteristics at baseline

\begin{tabular}{|c|c|c|c|}
\hline Characteristic & Placebo $(n=43)$ & Alirocumab 75 mg/150 mg q2w $(\mathrm{n}=40)$ & Total $(\mathrm{n}=83)$ \\
\hline Age, yr & $60.2 \pm 9.4$ & $60.8 \pm 9.4$ & $60.5 \pm 9.3$ \\
\hline \multicolumn{4}{|l|}{ Age group, yr } \\
\hline$<45$ & $2(4 \cdot 7)$ & $3(7.5)$ & $5(6.0)$ \\
\hline$\geq 45$ to $<65$ & $26(60.5)$ & $22(55 \cdot 0)$ & $48(57.8)$ \\
\hline$\geq 65$ to $<75$ & $13(30.2)$ & $12(30.0)$ & $25(30.1)$ \\
\hline$\geq 75$ & $2(4 \cdot 7)$ & $3(7 \cdot 5)$ & $5(6.0)$ \\
\hline \multicolumn{4}{|l|}{ Sex } \\
\hline Male & $35(81.4)$ & $32(80.0)$ & $67(80.7)$ \\
\hline Female & $8(18.6)$ & $8(20.0)$ & $16(19 \cdot 3)$ \\
\hline Weight, kg & $71.03 \pm 13.75$ & $72.48 \pm 12.67$ & $71.73 \pm 13.18$ \\
\hline $\mathrm{BMI}, \mathrm{kg} / \mathrm{m}^{2}$ & $25.91 \pm 3.67$ & $25.88 \pm 3 \cdot 35$ & $25 \cdot 90 \pm 3 \cdot 50$ \\
\hline \multicolumn{4}{|l|}{ BMI group, $\mathrm{kg} / \mathrm{m}^{2}$} \\
\hline$<30$ & $39(90.7)$ & $35(87 \cdot 5)$ & $74(89.2)$ \\
\hline$\geq 30$ & $4(9 \cdot 3)$ & $5(12.5)$ & $9(10.8)$ \\
\hline CV history/risk factors & $43(100.0)$ & $40(100.0)$ & $83(100.0)$ \\
\hline CHD & $40(93.0)$ & $40(100)$ & $80(96.4)$ \\
\hline Coronary revascularization procedures & $33(76.7)$ & $29(72.5)$ & $62(74 \cdot 7)$ \\
\hline Acute MI & $16(37.2)$ & $16(40.0)$ & $32(38.6)$ \\
\hline Unstable angina & $9(20.9)$ & $16(40.0)$ & $25(30.1)$ \\
\hline Silent MI & $7(16.3)$ & $4(10.0)$ & $11(13 \cdot 3)$ \\
\hline Other clinically significant CHD & $20(46.5)$ & $16(40.0)$ & $36(43 \cdot 4)$ \\
\hline $\begin{array}{l}\text { CHD associated with } \geq 1 \text { comorbidity and/or } \\
\text { associated with other CVD }\end{array}$ & $29(67 \cdot 4)$ & $29(72.5)$ & $58(69 \cdot 9)$ \\
\hline CHD risk equivalents & $9(20.9)$ & $5(12.5)$ & $14(16.9)$ \\
\hline Ischemic stroke & $4(9 \cdot 3)$ & $1(2.5)$ & $5(6.0)$ \\
\hline Moderate CKD & $4(9 \cdot 3)$ & $4(10.0)$ & $8(9.6)$ \\
\hline $\begin{array}{l}\text { Known history of } \mathrm{T} 2 \mathrm{DM} \text { and } \geq 2 \text { addition- } \\
\text { al risk factors }\end{array}$ & $1(2.3)$ & o & $1(1.2)$ \\
\hline $\begin{array}{l}\text { At least } 2 \text { or more CHD risk equivalents or } \\
\text { one CHD risk equivalent associated with } \\
\text { hypertension or } \mathrm{T} 2 \mathrm{DM}\end{array}$ & $8(18.6)$ & $3(7.5)$ & $11(13 \cdot 3)$ \\
\hline Prior history of MI or ischemic stroke & $27(62.8)$ & $24(60.0)$ & $51(61.4)$ \\
\hline \multicolumn{4}{|l|}{ Intensity of statins prescribed } \\
\hline High & $24(55 \cdot 8)$ & $22(55 \cdot 0)$ & $46(55 \cdot 4)$ \\
\hline Low/moderate & $19(44 \cdot 2)$ & $18(45 \cdot 0)$ & $37(44 \cdot 6)$ \\
\hline Any LLT other than statins & $14(32.6)$ & $14(35 \cdot 0)$ & $28(33.7)$ \\
\hline Ezetimibe & $4(9.3)$ & $7(17 \cdot 5)$ & $11(13 \cdot 3)$ \\
\hline
\end{tabular}

Values are presented as mean \pm SD or number (\%).

q2w, every 2 weeks; BMI, body mass index; CV, cardiovascular; CHD, coronary heart disease; MI, myocardial infarction; CVD, cardiovascular disease; CKD, chronic kidney disease; T2DM, type 2 diabetes mellitus; LLT, lipid lowering therapy. 
Table 2. Baseline lipid profile of study patients

\begin{tabular}{lccr}
\hline Variable & Placebo $(\mathrm{n}=43)$ & Alirocumab $75 \mathrm{mg} / 150 \mathrm{mg}$ q2W $(\mathrm{n}=40)$ & Total $(\mathrm{n}=83)$ \\
\hline LDL-C, mg/dL & $95.1 \pm 27.0$ & $90.7 \pm 22.9$ & $93.0 \pm 25.0$ \\
Non-HDL-C, mg/dL & $124.4 \pm 32.6$ & $117.4 \pm 27.0$ & $121.0 \pm 30.1$ \\
HDL-C, $\mathrm{mg} / \mathrm{dL}$ & $48.5 \pm 13.1$ & $45.9 \pm 10.6$ & $47.3 \pm 11.9$ \\
Fasting TG, mg/dL & $146.2 \pm 67.9$ & $144.2 \pm 117.1$ & $145.3 \pm 94.3$ \\
TC, $\mathrm{mg} / \mathrm{dL}$ & $172.9 \pm 28.4$ & $163.3 \pm 28.0$ & $168.3 \pm 28.4$ \\
Lp(a), mg/dL & $43.4 \pm 36.4$ & $37.0 \pm 36.8$ & $40.4 \pm 36.5$ \\
Apo Al, mg/dL & $136.2 \pm 25.1$ & $134.0 \pm 16.9$ & $135.1 \pm 21.5$ \\
Apo B, $\mathrm{mg} / \mathrm{dL}$ & $84.6 \pm 18.2$ & $79.3 \pm 14.7$ & $82.1 \pm 16.7$ \\
\hline
\end{tabular}

Values are presented as mean $\pm \mathrm{SD}$.

q2w, every 2 weeks; LDL-C, low density lipoprotein cholesterol; HDL-C, high density lipoprotein cholesterol; TG, triglyceride; TC, total cholesterol; Lp(a), lipoprotein(a); Apo A1, apolipoprotein A1; Apo B, apolipoprotein B.

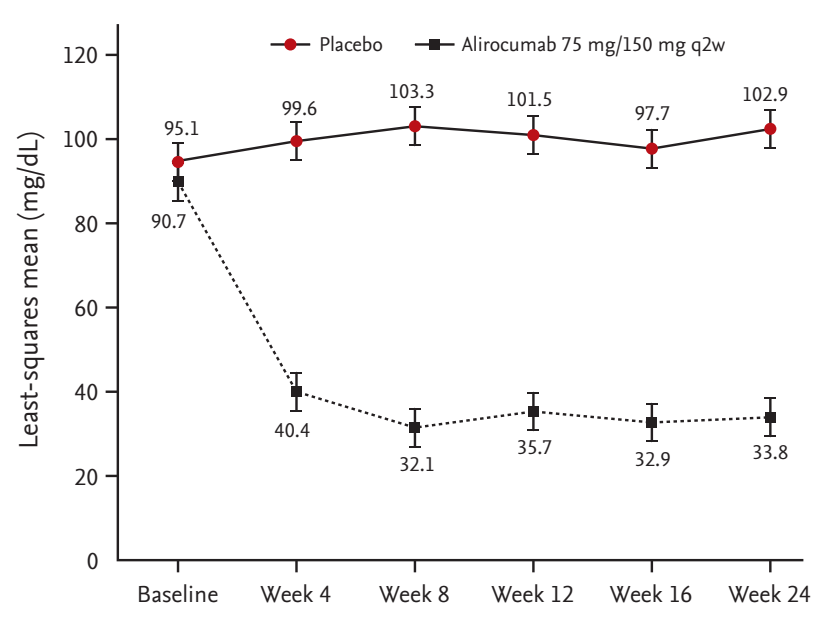

Figure 2. Calculated low density lipoprotein cholesterol levels (least-squares mean \pm standard error) in intent-to-treat population during treatment phase. q2w, every 2 weeks.

Eight treatment-emergent SAEs were experienced by five patients (12.5\%) from the alirocumab group. These SAEs were unstable angina, brachiocephalic artery stenosis, traumatic fracture, radius fracture (one patient each) and two ligament sprains and two muscle strains (one patient). Eight treatment-emergent SAEs were experienced by five patients (11.6\%) from the placebo group. These SAEs were road traffic accident and arterial injury (one patient); acute myocardial infarction on two occasions (one patient); unstable angina and peripheral arterial disease (one patient); pulmonary tuberculosis (one patient) and coronary artery stenosis (one patient). None of the reported SAEs were deemed related to study drug by both the investigator and the sponsor. No treat- ment-related discontinuation or death was reported due to TEAEs in either group.

\section{DISCUSSION}

The ODYSSEY KT study was the first to evaluate the safety and efficacy of alirocumab $75 \mathrm{mg}$ (with a provision to uptitrate the dose to $150 \mathrm{mg}$ ) every 2 weeks for 24 weeks in Korean patients with inadequately controlled hypercholesterolemia and at high risk of CVD. The main findings are as follows. (1) The percent change of LDL-C from baseline in the ITT population was $-65.7 \%$ in the alirocumab group and the difference of LDL-C versus placebo was $-76.7 \%(p<0.0001)$. (2) Alirocumab treatment additionally improved lipid parameters including HDL-C, non-HDL-C, TC, Lp(a), and Apo B versus placebo in the Korean cohort $(p<0.05)$. (3) Alirocumab did not induce higher rates of TEAEs and SAEs than placebo. None of the eight treatment-emergent SAEs in the alirocumab group, were deemed related to alirocumab.

In recent years, improvements in laboratory lipid measurements through $\mathrm{PCSK}_{9}$ inhibition have revealed to be associated with better clinical prognosis. Evolocumab reduced the risk of CV events (composite of CV death, myocardial infarction, stroke, hospitalization for unstable angina, or coronary revascularization) by $15 \%$ at 3 years of follow-up in FOURIER clinical trial [23]. Most recently in ODYSSEY outcomes trial, alirocumab proved $15 \%$ reduction in CV events (composite of CHD death, non-fatal myocardial infarction, fatal or non-fatal 
Table 3. Least squares mean differences (vs. placebo) in LDL-C and secondary lipid parameters in patients of alirocumab group in intent-to-treat population

\begin{tabular}{|c|c|c|c|c|c|}
\hline \multirow{2}{*}{ Lipid parameter } & \multicolumn{2}{|c|}{ \% Change at week 24 from baseline } & \multirow{2}{*}{$\begin{array}{c}\text { Difference of } \% \text { change } \\
\text { compared to placebo group }\end{array}$} & \multirow{2}{*}{$95 \%$ CI } & \multirow{2}{*}{$p$ value } \\
\hline & Placebo $(n=43)$ & Alirocumab $(\mathrm{n}=40)$ & & & \\
\hline LDL-C & $11.1 \pm 4.2$ & $-65 \cdot 7 \pm 4 \cdot 4$ & $-76.7 \pm 6.1$ & -88.9 to -64.6 & $<0.0001$ \\
\hline Non-HDL-C & $8.1 \pm 3.1$ & $-54.2 \pm 3.2$ & $-62.4 \pm 4.5$ & -71.4 to -53.4 & $<0.0001$ \\
\hline HDL-C & $7 \cdot 5 \pm 2.9$ & $16.6 \pm 3.0$ & $9.0 \pm 4.2$ & 0.7 to 17.4 & 0.0344 \\
\hline Fasting TG & $-1.6 \pm 4.9$ & $-8.7 \pm 5.0$ & $-7.1 \pm 7.0$ & -20.8 to 6.5 & 0.3048 \\
\hline $\mathrm{TC}$ & $7.1 \pm 2.3$ & $-34 \cdot 9 \pm 2.4$ & $-41.9 \pm 3.4$ & -48.6 to $-35 \cdot 3$ & $<0.0001$ \\
\hline $\mathrm{Lp}(\mathrm{a})$ & $4.8 \pm 5 \cdot 4$ & $-36.1 \pm 5.5$ & $-40.9 \pm 7.7$ & -56.1 to -25.8 & $<0.0001$ \\
\hline Apo A1 & $6.2 \pm 2.1$ & $3.4 \pm 2.1$ & $-2.8 \pm 3.0$ & -8.8 to 3.1 & 0.3479 \\
\hline Apo B & $8.1 \pm 3.1$ & $-48.9 \pm 3.2$ & $-57.0 \pm 4.5$ & -66.0 to -48.1 & $<0.0001$ \\
\hline
\end{tabular}

Values are presented as mean $\pm \mathrm{SE}$.

LDL-C, low density lipoprotein cholesterol; CI, confidence interval; HDL-C, high density lipoprotein cholesterol; TG, triglyceride; TC, total cholesterol; Lp(a), lipoprotein(a); Apo A1, apolipoprotein A1; Apo B, apolipoprotein B.

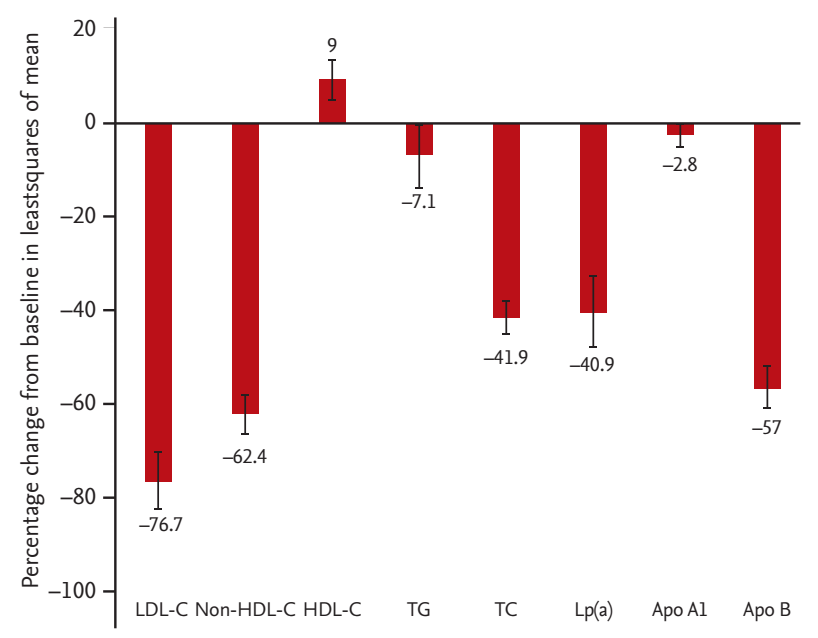

Figure 3. Least squares mean difference (vs. placebo) in low density lipoprotein cholesterol (LDL-C) and secondary lipid parameters in patients in the alirocumab group in intentto-treat population. LDL-C levels given were calculated values. Triglyceride levels were ascertained under fasting conditions. HDL-C, high density lipoprotein cholesterol; TG, triglyceride; TC, total cholesterol; Lp(a), lipoprotein(a); Apo A1, apolipoprotein A1; Apo B, apolipoprotein B.

ischemic stroke, or unstable angina requiring hospitalization) and appeared associated with 15\% lower rate of all-cause death at 4 years of follow-up among patients with "recent acute coronary syndrome" defined as an index hospitalization for acute myocardial infarction or unstable angina 1 to 12 months before the enrollment [24]. In summary, it can be implicated that the improve-

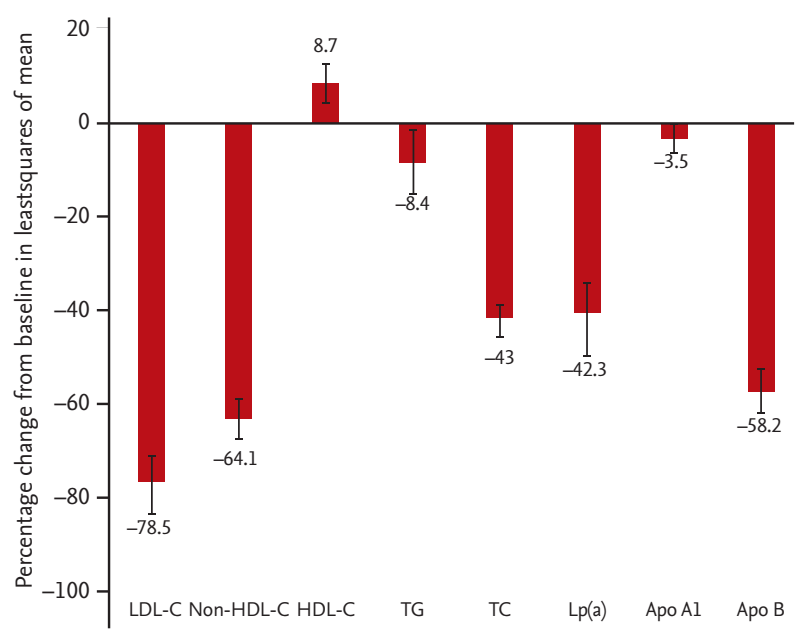

Figure 4. Least squares mean difference (vs. placebo) in low density lipoprotein cholesterol (LDL-C) and secondary lipid parameters in patients in the alirocumab group in modified intent-to-treat population. LDL-C levels given were calculated values. Triglyceride levels were ascertained under fasting conditions. HDL-C, high density lipoprotein cholesterol; TG, triglyceride; TC, total cholesterol; Lp(a), lipoprotein(a); Apo A1, apolipoprotein A1; Apo B, apolipoprotein B.

ments in LDL-C and related lipid parameters observed in current study might lead to clinical benefits in CV morbidity and mortality among Korean population. It requires further investigation.

There were several findings in the current Korean cohort that were unique relative to the entire ODYSSEY KT population [22]. The use of high-intensity statins was 
lower in the Korean cohort in comparison to the entire KT population (55.4\% vs. $72.4 \%)$. In contrast, the use of LLTs other than statins were higher in the Korean cohort that in the study population (33.7\% vs. $23.1 \%$ ). Additionally, baseline Lp(a) and fasting TG levels were higher in the Korean cohort. Compared to the entire ODYSSEY KT study population, nominally larger percent change in LDL-C from baseline in the alirocumab group appeared at week 12 (-62.3\% vs. $-57.9 \%)$ and was maintained week $24(-65.7 \%$ vs. $-57.1 \%)$ in the Korean cohort. Similarly, among alirocumab group, the proportion of patients achieving LDL-C values $<70 \mathrm{mg} / \mathrm{dL}$ (92.0\% vs. $85.8 \%$ ) and the proportion of patients with two consecutive LDL-C values $<15 \mathrm{mg} / \mathrm{dL}$ (10.0\% vs. $9.3 \%$ ) or $<25 \mathrm{mg} / \mathrm{dL}$ (37.5\% vs. $27.8 \%)$ were nominally higher in the Korean cohort. In short, LDL-C reduction through alirocumab treatment in Korean cohort appeared at least consistent with the results from whole ODYSSEY KT cohort. With regards to safety data, a smaller proportion of overall patients in the Korean cohort displayed TEAEs in comparison to the ODYSSEY KT study population (48.2\% vs. 60.3\%). Similarly, the incidence of TEAEs in the alirocumab-treated patients was lower in the Korean cohort in comparison to those in the ODYSSEY KT population (45.0\% vs. 58.8\%). In addition, neither death nor permanent discontinuation due to TEAE were observed in the Korean cohort, whereas one death (influenza A infection) and three permanent discontinuations due to TE$\mathrm{AEs}$ (one in placebo group and two in alirocumab group) were reported in the Taiwanese study population.

The results show that, with similar design and inclusion criteria, Korean patients exhibited consistent LDL-C reduction with previous ODYSSEY trials. In the ODYSSEY COMBO I and II studies, treatment with addon alirocumab to background statin therapy reduced LDL-C levels by at least $48.2 \%[14,17]$. In the ODYSSEY OPTIONS I and II studies, add-on alirocumab to atorvastatin 20 or $40 \mathrm{mg}$ resulted in a reduction of LDL-C levels of $44.1 \%$ and $54 \%$, respectively [13], while add-on alirocumab to rosuvastatin 10 or $20 \mathrm{mg}$ resulted in a reduction of LDL-C levels of $50.6 \%$ and $36.3 \%$, respectively [15]. In patients with heterozygous familial hypercholesterolemia (heFH), alirocumab treatment reduced LDL-C levels by at least 51.4\% [16]. In patients with statin intolerance, alirocumab treatment reduced LDL-C levels by $45 \%$ [18]. Another study reported that $150 \mathrm{mg}$ of
Table 4. Adverse events and laboratory evaluations in safety population (with incidence of $\geq 5.0 \%$ in alirocumab group)

\begin{tabular}{llc}
\hline Variable & $\begin{array}{c}\text { Placebo } \\
(\mathrm{n}=43)\end{array}$ & $\begin{array}{c}\text { Alirocumab 75 } \\
\mathrm{mg} / 150 \mathrm{mg} \text { q2W } \\
(\mathrm{n}=40)\end{array}$ \\
\hline Patients with any TEAE & $22(51.2)$ & $18(45.0)$ \\
$\begin{array}{l}\text { Patients with any } \\
\text { treatment-emergent SAE }\end{array}$ & $5(11.6)$ & $5(12.5)$ \\
\hline Classification according to SOC and PT & \\
\hline Nervous system disorders & $2(4.7)$ & $5(12.5)$ \\
\hline Headache & 0 & $4(10.0)$ \\
\hline Dizziness & 0 & $1(2.5)$ \\
\hline Infections and infestations & $6(14.0)$ & $4(10.0)$ \\
\hline Nasopharyngitis & $2(4.7)$ & $3(7.5)$ \\
\hline URTI & 0 & $1(2.5)$ \\
\hline Injury, fall, and procedural & $3(7.0)$ & $4(10.0)$ \\
complications & & $1(2.5)$ \\
\hline Fall & $1(2.3)$ & $1(2.5)$ \\
\hline Facial bones fracture & 0 & $1(2.5)$ \\
\hline Gastrointestinal disorders & $5(11.6)$ & $2(5.0)$ \\
\hline Chronic gastritis & 0 & $1(2.5)$ \\
\hline Dyspepsia & 0 & $1(2.5)$ \\
\hline Skin and subcutaneous & $1(2.3)$ & $2(5.0)$ \\
tissue disorders & & \\
\hline Eczema & 0 & \\
\hline Rash & 0 & \\
\hline
\end{tabular}

Values are presented as number (\%).

q2w, every 2 weeks; TEAE, treatment-emergent adverse event; SAE, serious adverse event; SOC, system organ class; PT, preferred term; URTI, upper respiratory tract infection.

alirocumab every 2 weeks induced $-62.0 \%$ of change in LDL-C level at week 24 from baseline [19]. Considering that only three out of 40 patients were uptitrated to 150 $\mathrm{mg}$ in Korean cohort, it might suggest that $75 \mathrm{mg}$ every 2 weeks is enough for the majority of Korean dyslipidemic patients. In studies on Japanese patients (with or without heFH), alirocumab treatment reduced LDL-C levels by at least $40 \%[20,21]$. Similar to the results observed in this Korean population, a pooled analysis of eight ODYSSEY phase 3 trials $(n=4,629)$ indicated that alirocumab provided consistent reductions in LDL-C regardless of the statin dose and type [25]. In the ODYSSEY LONG TERM trial $(\mathrm{n}=2,341)$, TEAEs were observed 
in $81 \%$ of alirocumab-treated patients [19], which is more than that observed in the Korean cohort (45\%). The most common TEAEs in the ODYSSEY LONG TERM trial of alirocumab-treated patients were general allergic reactions (10.1\%) and local injection-site reactions (5.9\%) [19], while the most common TEAEs in the Korean cohort were headache (10.0\%) and nasopharyngitis (7.5\%) (Table 4).

While the patients in the ODYSSEY KT study were receiving background statin therapy, it is important to note that high-dose statins are known to upregulate PCSK9 expression [26] and could therefore interfere with PCSK9 inhibitors.

Although several reports suggested that low-to-moderate dose statins are sufficient in Korean population [27,28], it still appears to be inadequate in achieving the goal of LDL-C $<70 \mathrm{mg} / \mathrm{dL}$ in patients with very high CV risk [6-8]. Further investigations are needed to assess the necessity of more aggressive treatment policy including combination of alirocumab for Korean dyslipidemic patients.

There are several limitations to this study. The relatively small number of patients $(n=40)$ in the alirocumab group may not be representative of the relevant Korean population. There was a 4:1 ratio of males to females in the study, and very few patients were $<45$ or $>75$ years. As this study recruited patients already on statins or other LLTs, it is not known how the Korean population would respond to alirocumab as monotherapy. The small number of patients and the limited trial period may be insufficient to capture all relevant AEs.

In conclusion, this study demonstrated that alirocum$\mathrm{ab}$ showed significantly greater efficacy in reduction of LDL-C levels, as well as improvements in HDL-C, nonHDL-C, TC, Lp(a), and Apo B than placebo in Korean patients. In addition, alirocumab was generally well tolerated. These results indicate that Korean dyslipidemic patients could achieve higher efficacy in LDL-C reduction with alirocumab augmentation than with conventional LLTs only.

\section{KEY MESSAGE}

1. Alirocumab add on treatment can make an additional low density lipoprotein cholesterol level reduction up to $65 \%$ to $75 \%$ compared with statin only therapy.

2. Alirocumab treatment additionally improved lipid parameters including high density lipoprotein cholesterol (HDL-C), non-HDL-C, total cholesterol, lipoprotein(a), and apolipoprotein B versus placebo in the Korean patients.

3. Although alirocumab showed stronger effect on lipid modification than placebo, alirocumab did not induce higher rates of treatment-emergent adverse events and serious adverse events than statin only therapy.

\section{Conflict of interest}

This study was funded by Sanofi and Regeneron Pharmaceuticals, Inc.

\section{Acknowledgments}

This study was supported by Sanofi and Regeneron in data collection and analysis, assistance with statistical analysis, and critical review of the article. Medical writing and editorial support in the preparation of this publication was provided by Satyendra Shenoy from Describe Scientific Writing \& Communications and Derek Ho who were paid for by Sanofi and Anahita Gouri and Rohan Mitra from Sanofi. The authors, individually and collectively, are responsible for all content and editorial decisions. Chang-Wook Nam, Dong-Soo Kim, ChongJin Kim received payment from neither Sanofi nor Regeneron Pharmaceuticals, Inc. directly or indirectly (through a third party) related to the development/presentation of this publication.

\section{REFERENCES}

1. Jeong JS, Kwon HS. Prevalence and clinical characteristics of dyslipidemia in Koreans. Endocrinol Metab (Seoul) 2017;32:30-35.

2. Lim D, Ha M, Song I. Trends in the leading causes of death in Korea, 1983-2012. J Korean Med Sci 2014;29:1597-1603.

3. Korea Centers for Disease Control and Prevention. Program for prevention and management of a cardio-cerebrovascular disease [Internet]. Cheongju (KR): 
KCDC, 2017 [cited 2018 Aug 11]. Available from: http:// www.cdc.go.kr/CDC/eng/contents/CdcEngContentView.jsp?cid=74261\&menuIds=HOMEoo2-MNU0576MNU0582.

4. Lim S, Shin $\mathrm{H}$, Song JH, et al. Increasing prevalence of metabolic syndrome in Korea: the Korean National Health and Nutrition Examination Survey for 1998-2007. Diabetes Care 2011;34:1323-1328.

5. Korean Society of Lipidology and Atherosclerosis. Dyslipidemia fact sheet in Korea [Internet]. Seoul (KR): Korean Society of Lipidology and Atherosclerosis, c2015 [cited 2018 Aug 11]. Available from: http://www.lipid.or.kr/file/ Dyslipidemia\%2ofact\%2osheet_eng_Final.pdf.

6. Authors/Task Force Members, Catapano AL, Graham I, et al. 2016 ESC/EAS guidelines for the management of dyslipidaemias: the task force for the management of dyslipidaemias of the European Society of Cardiology (ESC) and European Atherosclerosis Society (EAS) developed with the special contribution of the European Association for Cardiovascular Prevention \& Rehabilitation (EACPR). Atherosclerosis 2016;253:281-344.

7. Jacobson TA, Ito MK, Maki KC, et al. National lipid association recommendations for patient-centered management of dyslipidemia: part 1: full report. J Clin Lipidol 2015;9:129-169.

8. Committee for the Korean Guidelines for the Management of Dyslipidemia. 2015 Korean guidelines for the management of dyslipidemia: executive summary (English translation). Korean Circ J 2016;46:275-306.

9. Park JE, Chiang CE, Munawar M, et al. Lipid-lowering treatment in hypercholesterolaemic patients: the CEPHEUS Pan-Asian survey. Eur J Prev Cardiol 2012;19:781-794.

10. Seidah NG. New developments in proprotein convertase subtilisin-kexin 9's biology and clinical implications. Curr Opin Lipidol 2016;27:274-281.

11. Landmesser U, Chapman MJ, Farnier M, et al. European Society of Cardiology/European Atherosclerosis Society Task Force consensus statement on proprotein convertase subtilisin/kexin type 9 inhibitors: practical guidance for use in patients at very high cardiovascular risk. Eur Heart J 2017;38:2245-2255.

12. Writing Committee, Lloyd-Jones DM, Morris PB, et al. 2016 ACC expert consensus decision pathway on the role of non-statin therapies for LDL-cholesterol lowering in the management of atherosclerotic cardiovascular disease risk: a report of the American College of Cardiology task force on clinical expert consensus documents. J Am Coll Cardiol 2016;68:92-125.

13. Bays H, Gaudet D, Weiss R, et al. Alirocumab as add-on to atorvastatin versus other lipid treatment strategies: ODYSSEY OPTIONS I randomized trial. J Clin Endocrinol Metab 2015;100:3140-3148.

14. Cannon CP, Cariou B, Blom D, et al. Efficacy and safety of alirocumab in high cardiovascular risk patients with inadequately controlled hypercholesterolaemia on maximally tolerated doses of statins: the ODYSSEY COMBO II randomized controlled trial. Eur Heart J 2015;36:1186-1194.

15. Farnier M, Jones P, Severance R, et al. Efficacy and safety of adding alirocumab to rosuvastatin versus adding ezetimibe or doubling the rosuvastatin dose in high cardiovascular-risk patients: the ODYSSEY OPTIONS II randomized trial. Atherosclerosis 2016;244:138-146.

16. Kastelein JJ, Ginsberg HN, Langslet G, et al. ODYSSEY FH I and FH II: 78 week results with alirocumab treatment in 735 patients with heterozygous familial hypercholesterolaemia. Eur Heart J 2015;36:2996-3003.

17. Kereiakes DJ, Robinson JG, Cannon CP, et al. Efficacy and safety of the proprotein convertase subtilisin/kexin type 9 inhibitor alirocumab among high cardiovascular risk patients on maximally tolerated statin therapy: the ODYSSEY COMBO I study. Am Heart J 2015;169:906-915.

18. Moriarty PM, Thompson PD, Cannon CP, et al. Efficacy and safety of alirocumab vs ezetimibe in statin-intolerant patients, with a statin rechallenge arm: the ODYSSEY ALTERNATIVE randomized trial. J Clin Lipidol 2015;9:758-769.

19. Robinson JG, Farnier M, Krempf M, et al. Efficacy and safety of alirocumab in reducing lipids and cardiovascular events. N Engl J Med 2015;372:1489-1499.

20. Teramoto T, Kobayashi M, Tasaki H, et al. Efficacy and safety of alirocumab in Japanese patients with heterozygous familial hypercholesterolemia or at high cardiovascular risk with hypercholesterolemia not adequately controlled with statins: ODYSSEY Japan randomized controlled trial. Circ J 2016;80:1980-1987.

21. Teramoto T, Kobayashi M, Uno K, et al. Efficacy and safety of alirocumab in Japanese subjects (phase 1 and 2 studies). Am J Cardiol 2016;118:56-63.

22. Koh KK, Nam CW, Chao TH, et al. A randomized trial evaluating the efficacy and safety of alirocumab in South Korea and Taiwan (ODYSSEY KT). J Clin Lipidol 
2018;12:162-172.

23. Sabatine MS, Giugliano RP, Keech AC, et al. Evolocumab and clinical outcomes in patients with cardiovascular disease. N Engl J Med 2017;376:1713-1722.

24. American College of Cardiology. ACC.18 Joint ACC/JACC late-breaking clinical trials [Internet]. Washington, DC: American College of Cardiology, c2018 [cited 2018 Aug 11]. Available from: https://accscientificsession.acc.org/ features/2018/03/video-sanofi-regeneron.

25. Catapano AL, Lee LV, Louie MJ, Thompson D, Bergeron J, Krempf M. Efficacy of alirocumab according to background statin type and dose: pooled analysis of 8 ODYS-
SEY phase 3 clinical trials. Sci Rep 2017;7:45788.

26. Dubuc G, Chamberland A, Wassef H, et al. Statins upregulate $\mathrm{PCSK} 9$, the gene encoding the proprotein convertase neural apoptosis-regulated convertase-1 implicated in familial hypercholesterolemia. Arterioscler Thromb Vasc Biol 2004;24:1454-1459.

27. Kwon JE, Kim Y, Hyun S, et al. Cholesterol lowering effects of low-dose statins in Korean patients. J Lipid Atheroscler 2014;3:21-28.

28. Kim SK. Re-evaluation of efficacy of moderate-intensity statins in Korean patients with type 2 diabetes mellitus. Diabetes Metab J 2017;41:20-22. 


\section{Supplementary Table 1. Exclusion criteria}

$1 \quad$ Patients without established CHD or CHD risk equivalents

2 LDL-C,70 mg/dL at the screening visit in patients with a history of documented CVD

3 LDL-C, $100 \mathrm{mg} / \mathrm{dL}$ at the screening visit in patients without history of documented CVD

4 Not on a stable dose of lipid lowering therapy (including statin) for $\geq 4$ weeks before the screening visit or between screening and randomization visits

5 Currently taking a statin that is not atorvastatin, rosuvastatin, or simvastatin

6 Atorvastatin, rosuvastatin, or simvastatin is not taken daily or not taken at a registered dose

7 Daily dose above atorvastatin $80 \mathrm{mg}$, rosuvastatin $20 \mathrm{mg}$, or simvastatin $40 \mathrm{mg}$

8 Use of fibrates, other than fenofibrate in the past 4 weeks before screening visit or between screening and randomization visits

9 Use of nutraceutical products or over-the-counter therapies that may affect lipids which have not been at a stable dose/ amount for $\geq 4$ weeks before the screening visit or between screening and randomization visits

10 Use of red yeast rice products within 4 weeks of the screening visit or between screening and randomization visits

11 Patient who has received plasmapheresis treatment within 2 months before the screening visit or has plans to receive this during the study

12 History of a MI, unstable angina leading to hospitalization, CABG, PCI, uncontrolled cardiac arrhythmia, carotid surgery or stenting, stroke, transient ischemic attack, carotid revascularization, endovascular procedure, or surgical intervention for peripheral vascular disease within 3 months before the screening visit

13 Planned to undergo scheduled PCI or CABG, or carotid or peripheral revascularization, during the study

14 Systolic blood pressure (160 $\mathrm{mmHg}$ ) or diastolic blood pressure (100 $\mathrm{mmHg}$ ) at screening visit or randomization visit

15 History of New York Heart Association Class III or IV heart failure within the past 12 months

16 Known history of hemorrhagic stroke

17 Age, 18 years or legal age of majority at the screening visit, whichever is greater

18 Patients not previously instructed on a cholesterol-lowering diet before the screening visit

19 Newly diagnosed (within 3 months before randomization visit) or poorly controlled (HbA1c $>9 \%$ at the screening visit) diabetes

20 Presence of any clinically significant uncontrolled endocrine disease known to influence serum lipids or lipoproteins

21 History of bariatric surgery within 12 months before the screening visit

22 Unstable weight defined by a variation $>5 \mathrm{~kg}$ within 2 months before the screening visit

23 Known history of homozygous or heterozygous familial hypercholesterolemia

24 Known history of loss of function of $\mathrm{PCSK}_{9}$ (i.e., genetic mutation or sequence variation)

25 Use of systemic corticosteroids, unless used as replacement therapy for pituitary/adrenal disease with a stable regimen for at least 6 weeks before randomization

26 Use of continuous estrogen or testosterone hormone replacement therapy unless the regimen has been stable in the past 6 weeks before the screening visit and no plans to change the regimen during the study

27 History of cancer within the past 5 years, except for adequately treated basal cell skin cancer, squamous cell skin cancer, or in situ cervical cancer

28 Known history of a positive HIV test

29 Patient who has taken any investigational drugs other than the alirocumab training placebo kits within 1 month or 5 half-lives, whichever is longer

30 Patient who has been previously treated with at least 1 dose of alirocumab or any other anti-PCSK9 monoclonal antibody in other clinical trials

31 Patient who withdraws consent during the screening period (patient who is not willing to continue or fails to return) 


\section{Supplementary Table 1. Continued}

32 Conditions/situations such as:

Any clinically significant abnormality identified at the time of screening that in the judgment of the investigator or any sub-investigator would preclude safe completion of the study or constrain endpoints assessment such as major systemic diseases or patients with a short life expectancy.

Considered by the investigator or any sub-investigator as inappropriate for this study for any reason, e.g.,

Deemed unable to meet specific protocol requirements, such as scheduled visits

Deemed unable to administer or tolerate long-term injections as per the patient or the investigator

Investigator or any sub-investigator, pharmacist, study coordinator, other study staff, or relative thereof directly involved in the conduct of the protocol, etc.

Presence of any other conditions (e.g., geographic, social), actual or anticipated, that the investigator feels would restrict or limit the patient's participation for the duration of the study

33 Laboratory findings during the screening period:

Positive test for hepatitis B surface antigen or hepatitis C antibody (confirmed by reflexive testing)

Positive serum $\beta$-human chorionic gonadotropin or urine pregnancy test in women of childbearing potential

Triglycerides $>400 \mathrm{mg} / \mathrm{dL}$ (1 repeat laboratory is allowed)

eGFR $<30 \mathrm{~mL} / \mathrm{min} / 1.73 \mathrm{~m}^{2}$

ALT or AST $>3 \times$ ULN ( 1 repeat laboratory is allowed)

$\mathrm{CPK}>3 \times \mathrm{ULN}$ (1 repeat laboratory is allowed)

TSH $<$ lower limit of normal or $>$ ULN (1 repeat laboratory is allowed)

34 All contraindications to the background therapies or warning/precaution of use (when appropriate) as displayed in the respective National Product Labeling

35 Known hypersensitivity to monoclonal antibody or any component of the drug products

36 Pregnant or breastfeeding women

37 Women of childbearing potential not protected by highly effective method(s) of birth control and/or who are unwilling or unable to be tested for pregnancy

CHD, coronary heart disease; LDL-C, low density lipoprotein cholesterol; CVD, cardiovascular disease; MI, myocardial infarction; CABG, coronary artery bypass graft; PCI, percutaneous coronary intervention; HbA1c, glycated hemoglobin; PCSK9, proprotein convertase subtilisin/kexin type 9; HIV, human immunodeficiency virus; eGFR, estimated glomerular filtration rate; ALT, alanine aminotransferase; AST, aspartate transferase; ULN, upper limit of normal; CPK, creatine phosphokinase; TSH, thyroid stimulating hormone. 
Supplementary Table 2. Least squares mean differences (vs. placebo) in LDL-C and secondary lipid parameters in patients of alirocumab group in modified intent-to-treat population

\begin{tabular}{|c|c|c|c|c|c|}
\hline \multirow[b]{2}{*}{ Lipid parameter } & \multicolumn{2}{|c|}{ \% Change at week 24 from baseline } & \multirow{2}{*}{$\begin{array}{l}\text { Difference of \% } \\
\text { change compared } \\
\text { to placebo group }\end{array}$} & \multirow[b]{2}{*}{$95 \% \mathrm{CI}$} & \multirow[b]{2}{*}{$p$ value } \\
\hline & $\begin{array}{l}\text { Placebo } \\
(n=43)\end{array}$ & $\begin{array}{l}\text { Alirocumab } \\
(\mathrm{n}=40)\end{array}$ & & & \\
\hline LDL-C & $11.1 \pm 4.2$ & $-67 \cdot 3 \pm 4 \cdot 4$ & $-78.5 \pm 6.1$ & -90.6 to -66.3 & $<0.0001$ \\
\hline non-HDL-C & $8.2 \pm 3.1$ & $-55 \cdot 9 \pm 3 \cdot 2$ & $-64.1 \pm 4.4$ & -72.9 to -55.3 & $<0.0001$ \\
\hline HDL-C & $8.1 \pm 2.9$ & $16.9 \pm 3.0$ & $8.7 \pm 4.2$ & 0.4 to 17.1 & 0.0412 \\
\hline Fasting TG & $-0.05 \pm 4.9$ & $-8.3 \pm 5.0$ & $-8.3 \pm 7.0$ & -22.0 to 5.3 & 0.2298 \\
\hline $\mathrm{TC}$ & $7.1 \pm 2.3$ & $-35 \cdot 9 \pm 2.4$ & $-43 \cdot 0 \pm 3 \cdot 3$ & -49.7 to -36.4 & $<0.0001$ \\
\hline $\mathrm{Lp}(\mathrm{a})$ & $5 \cdot 3 \pm 5 \cdot 7$ & $-37.0 \pm 5.6$ & $-42.3 \pm 8.0$ & -58.0 to -26.6 & $<0.0001$ \\
\hline Apo A1 & $7.2 \pm 2.1$ & $3.7 \pm 2.2$ & $-3 \cdot 5 \pm 3 \cdot 0$ & -9.6 to 2.5 & 0.2482 \\
\hline Apo B & $7.9 \pm 3.1$ & $-50.4 \pm 3.2$ & $-58.2 \pm 4.5$ & -67.2 to -49.2 & $<0.0001$ \\
\hline
\end{tabular}

Values are presented as mean $\pm \mathrm{SE}$.

LDL-C, low density lipoprotein cholesterol; CI, confidence interval; HDL-C, high density lipoprotein cholesterol; TG, triglyceride; TC, total cholesterol; Lp(a), lipoprotein(a); Apo A1, apolipoprotein A1; Apo B, apolipoprotein B. 